\title{
Mapping of Stellar Surfaces with Doppler and Zeeman Doppler Imaging
}

\author{
Andrew Collier Cameron \\ School of Physics and Astronomy, University of St Andrews, North \\ Haugh, St Andrews, SCOTLAND KY16 9SS
}

\begin{abstract}
Doppler imaging has been used to image dark spots on the surfaces of rapidly rotating, magnetically active stars for the last 20 years. More recently, methods have been developed for combining line-profile information from large numbers of spectral lines simultaneously. This has allowed starspot distributions to be mapped in sufficient detail to allow tracking of individual spots over several stellar rotations, delineating surface differential rotation patterns for a number of stars. Zeeman-Doppler imaging allows the creation of stellar magnetograms, which are providing the first insights into the 3D topology of stellar coronal magnetic fields. The advent of cryogenic infrared echelle spectrographs opens up exciting new possibilities for Doppler imaging in molecular lines of species such as $\mathrm{TiO}, \mathrm{OH}$, and $\mathrm{FeH}$.
\end{abstract}

\section{Doppler Imaging and Differential Rotation}

Stellar magnetic activity is driven by the interaction between differential rotation and convection in the outer envelopes of rapidly rotating solar-type stars. This activity manifests itself as coronal X-ray emission, chromospheric UV and optical line emission, and rotational modulation of the optical continuum by widespread starspot activity.

Since rotation is a key driver of this activity, many of the most active solartype stars spin fast enough that the rotational Doppler effect is the dominant line-broadening mechanism. In such rapidly rotating stars, different parts of the stellar surface become more or less separated in velocity space. Doppler imaging takes advantage of this separation, utilising spectra at resolving powers of order $R=50000$. Dark features on the stellar surface produce a flux deficit at all wavelengths except those of photospheric absorption lines at the local Doppler shift. As a result, bright pseudo-emission bumps appear in the rotationallybroadened profiles of nearly all the photospheric lines. These bumps drift across the profile from blue to red, following sinusoidal paths whose velocity amplitude is determined by the spot latitude, and whose period of recurrence is determined by the local rotation period at that latitude on the star.

This pattern of drifting features can be inverted tomographically to recover a map of the stellar surface. This inversion method, dubbed "Doppler imaging" in the pioneering paper by Vogt \& Penrod (1983) that first applied such techniques to spots on cool stars, was developed formally using a regularised inversion techniques by Goncharsky et al. (1977) using the regularisation method of Tikhonov (1963), and by Vogt, Penrod \& Hatzes (1987) using maximumentropy regularisation. Since then, several groups have developed codes that use 
either these methods or the "Occamian" singular-value decomposition method of Berdyugina (1998).

In the last two decades, many single and binary dwarf, subgiant and giant stars have been imaged by these groups. One of the more striking recent applications has been the use of starspots as tracers of stellar surface differential rotation patterns. This has been achieved via a variety of methods, ranging from latitude-by-latitude cross-correlation of images taken two or more rotations apart (Donati \& Cameron 1997), to more spohisticated modelling approaches in which the differential rotation model is built into the inversion code itself (Donati et al. 2000). The actual form of the differential rotation law has been verified via direct tracking of individual spot signatures in data space (Cameron, Donati \& Semel 2002). By and large, these studies have revealed that most dwarf and subgiant stars appear to have solar-like differential rotation, in the sense that the equator rotates faster than the poles, with the equator overtaking the polar regions on timescales of order tens to hundreds of days.

\section{Zeeman-Doppler Imaging}

Doppler imaging acquired a new dimension in the 1990s with the advent of high-precision fibre-fed echelle spectropolarimeters, in particular the portable unit deployed at the Anglo-Australian and Canada-France-Hawaii Telescopes by Semel et al. (1993). Using this instrument, Donati et al. (1997) demonstrated that, by creating an optimally-weighted sum of the Stokes V signatures in thousands of photospheric lines observed simultaneously, it was possible to detect and even map the vector magnetic field at the surface of an active star, with a surface resolution as small as a few degrees in some cases. This work has led Hussain et al. (2001) to investigate the three-dimensional topology of potential fields extrapolated from Zeeman-Doppler images of young pre-main sequence stars such as AB Dor and LQ Hya. Models in which these field structures are filled with X-ray emitting plasma in hydrostatic equilibrium are proving a powerful tool in interpreting the rotational modulation of coronal X-ray emission from these stars (Jardine et al. 2002). In the near future, new-generation spectropolarimeters such as CFHT/ESPADONS (Manset \& Donati 2003) and LBT/PEPSI-ICE (Strassmeier et al. 2003; Pallavicini et al. 2003) will enable Zeeman-Doppler imaging to be extended to fainter objects than have hitherto been accessible. In particular, it should be possible to map the magnetic fields of $\mathrm{T}$ Tauri stars and fully-convective $\mathrm{M}$ dwarfs, and so to discover the dynamo modes that are excited in the absence of a radiative-convective interface region.

\section{The Infrared Frontier}

One of the major deficiencies of Zeeman-Doppler imaging is that at optical wavelengths, the Zeeman signature of the magnetic field in the dark starspots is suppressed by the contrast between the low surface brightness in the spots and the bright photosphere. The advent of high-resolution infrared spectrometers such as GEMINI-S/PHOENIX (Hinkle et al. 1998) and VLT/CRIRES (Moorwood et al. 2003; Kaeufl et al. 2003) will enable us to produce Doppler images in lines of molecular species such as $\mathrm{TiO}, \mathrm{OH}$, and $\mathrm{FeH}$ that are only present in the 
spots. In the slightly longer term, theoretical and laboratory measurements of the Lande g-factors for these molecular transitions (e.g., Berdyugina \& Solanki 2002 ) should allow direct measurement of the field strengths in the spot umbrae themselves. This will represent a major step towards a full understanding of the magnetic flux budget in the photospheres, coronae, and winds of young stars, and its influence on the rotational evolution of both single and binary stars.

\section{References}

Berdyugina S. V., \& Solanki S. K. 2002, A\&A, 385, 701

Berdyugina S. V. 1998, A\&A, 338, 97

Collier Cameron A., Donati J.-F., \& Semel M. 2002, MNRAS, 330, 699

Donati J.-F., \& Collier Cameron A. 1997, MNRAS, 291, 1

Donati J.-F., Semel M., Carter B., Rees D. E., \& Collier Cameron A. 1997, MNRAS, 291,658

Donati J.-F., Mengel M., Carter B. D., Marsden S., Collier Cameron A., \& Wichmann R. 2000, MNRAS, 316, 699

Goncharsky A. V., Stepanov V. V., Khokhlova V. L., \& Yagola A. G. 1977, Soviet Astron. Lett., 3, 147

Hinkle K. H., et al. 1998, in Proceedings of the SPIE Vol. 3354, Infrared Astronomical Instrumentation, ed. A. M. Fowler, 810

Hussain G. A. J., Jardine M., \& Collier Cameron A. 2001, MNRAS, 322, 681

Jardine M., Wood K., Collier Cameron A., Donati J.-F., \& Mackay D. H. 2002, MNRAS, 336,1364

Kaeuf H., Moorwood A. F. M., \& Pirard J. 2003, in Proceedings of the SPIE Vol. 4843, Polarimetry in Astronomy, ed. S. Fineschi, 223

Manset N., \& Donati J. 2003, in Polarimetry in Astronomy, ed. S. Fineschi, Proceedings of the SPIE, 4843,425

Moorwood A. F. M., et al. 2003, in Proceedings of the SPIE Vol. 4841, Instrument Design and Performance for Optical/Infrared Ground-based Telescopes, ed. M. Iye, \& A. F. M. Moorwood, 1592

Pallavicini R., et al. 2003, in Proceedings of the SPIE Vol. 4841, Instrument Design and Performance for Optical/Infrared Ground-based Telescopes, ed. M. Iye, M. \& A. F. M. Moorwood, 1345

Semel M., Donati J.-F., \& Rees D. E. 1993, A\&A, 278, 231

Strassmeier K. G., et al. 2003, in Proceedings of the SPIE Vol. 4843, Polarimetry in Astronomy, ed. S. Fineschi, 180

Tikhonov A. N. 1963, Soviet Math. Dokl., 4, 1624

Vogt S. S., \& Penrod G. D. 1983, PASP, 95, 565

Vogt S. S., Penrod G. D., \& Hatzes A. P. 1987, ApJ, 321, 496 